\title{
Factores clave de la cadena logística del comercio exterior de un puerto mexicano: análisis a través de redes neuronales artificiales
}

\author{
Key factors of international trade logistics chain of the Mexican Port: \\ Analysis through artificial neural networks
}

\author{
América Ivonne Zamora Torres ${ }^{1 *}$ y Juan González García ${ }^{2}$ \\ ${ }^{1}$ Universidad Michoacana de San Nicolás de Hidalgo, México \\ ${ }^{2}$ Universidad de Colima, México
}

Recibido el 29 de abril de 2017; aceptado el 11 de mayo de 2018

Disponible en Internet el: 22 de noviembre de 2018

\section{Resumen}

Actualmente, el comercio internacional evoluciona, mostrando una mayor expansión de las cadenas de valor mundiales, que se han reforzado logrando ser más eficientes integrándose a la economía mundial con costos más bajos; gracias a los avances tecnológicos y la mejora de los procesos organizacionales, se ha aumentado la competitividad de los productos en los mercados mundiales. El objetivo de este trabajo es identificar los principales elementos de cinco factores clave de la cadena logística del comercio exterior del puerto de Lázaro Cárdenas y calcular el valor pronosticado para cada factor, identificando las áreas de oportunidad y proponer mejoras y adecuaciones. Para lo cual se utiliza la metodología de redes neuronales artificiales (RNA) a través del procesamiento perceptrón multicapa. Los resultados muestran la existencia de cinco variables, las cuales son altamente significativas, destacando de entre ellas la aduana que, obtuvo la ponderación más baja, lo que indica la necesidad de mejora de los procesos aduanales en el puerto, para hacerlo más eficiente.

Códigos JEL: C00, F13, F23, H1.

Palabras clave: Redes neuronales artificiales (RNA); cadena logística; comercio internacional; aduanas; costos; tiempo; almacenaje y transporte.

\footnotetext{
*Autor para correspondencia.

Correo electrónico: americazt@hotmail.com (A.Zamora Torres)

La revisión por pares es responsabilidad de la Universidad Nacional Autónoma de México.
} 


\begin{abstract}
Currently, international trade evolves showing different trends, such as the expansion of global value chains that have been reinforced to be more efficient achieving integrated into the world economy with lower costs; thanks to technological advances and improved organizational processes increasing product competitiveness in world markets. The objective of this study is to identify the main elements of five key factors in the logistics chain foreign trade port of Lazaro Cardenas and calculate the predicted value for each factor identifying areas of opportunity and propose improvements and adjustments. The methodological tool used are artificial neural networks (ANN) through the Multilayer Perceptron processing. The results show that the five variables are highly significant stressing that the office had the lowest weighting thus showing the need for improvement of customs processes in the port, to make it more efficient.
\end{abstract}

JEL Codes: C00, F13, F23, H11.

Keywords: Artificial Neural Network (ANN); supply chain; international trade; customs; cost; time; storage and transportation.

\title{
Introducción
}

Algunas de las características de la economía global están constituidas por los avances tecnológicos, así como por la existencia de consumidores con una cada vez mayor oferta de productos a su disposición. Esto hace, que las cadenas de suministro modernas se enfrenten a grandes retos para lograr un crecimiento sostenido y mantener sus posiciones de ventaja competitiva. Debido a lo anterior, se presenta una constante presión en los mercados para lograr reducir los costos y mejorar la calidad de los servicios proporcionados; de ahí la creciente importancia en la mejora continua de la cadena logística como factor clave para la competitividad de las empresas.

El concepto de una cadena logística en el comercio internacional, implica la integración de las diferentes partes o eslabones que conforman el proceso de comercialización, dentro de los cuales se identifican, entre los más importantes a: la infraestructura, los servicios de transporte, almacenaje, las tecnologías de la información utilizadas, los diferentes procesos logísticos inherentes a los movimientos de carga, así como los trámites oficiales requeridos. Todos estos eslabones operan entre los nodos de origen y destino de la cadena, propiciando la conectividad del comercio exterior, el cual incluye puntos intermedios en el corredor logístico.

De ahí la importancia de analizar los principales elementos que componen la cadena logística del comercio internacional con la finalidad de ver al interior de estos que variables son las más importantes dentro del sistema logístico, por lo cual el objetivo de la presente investigación es identificar los principales factores: aduanas, costos, tiempo, almacenaje y transporte, inherentes a la cadena logística del comercio exterior del puerto de Lázaro Cárdenas, así como determinar el valor pronosticado para cada uno de estos factores, a fin de demarcar las áreas de oportunidad en cada caso y proponer las mejoras y adecuaciones, para una mayor eficiencia futura.

Cabe señalar que se seleccionó el puerto de Lázaro Cárdenas como objeto de estudio debido a la gran importancia que, junto con el puerto de Manzanillo, Colima, tiene para México en materia de comercio exterior. En efecto, el puerto registra una fuerte inversión tanto pública como privada, lo que lo ubica actualmente como un puerto con infraestructura de vanguardia en constante expansión. Adicionalmente cabe señalar que los puertos mexicanos que conectan 
con Asia, cada vez más, irán cobrando una mayor importancia dada su posición estratégica en relación con los puertos de mayor productividad en el mundo (OMC, 2017).

La presente investigación se divide en seis secciones. La primera corresponde a la introducción, en la segunda se presentan los antecedentes del puerto de Lázaro Cárdenas y su cadena logística, en la tercera sección se aborda la metodología con la que se realizará el análisis a fin de lograr el objetivo de la investigación, en la cuarta sección se presentan los resultados derivados de la aplicación de la herramienta metodológica; en la quinta los pronósticos por variable y finalmente en la última sección, las conclusiones de la investigación.

\section{Antecedentes: el puerto de Lázaro Cárdenas y su cadena logística}

Actualmente la mayor parte del comercio internacional se lleva a cabo a través del transporte marítimo (OMC, 2015; 2017) por lo que hoy en día los puertos son los principales nodos en el intercambio comercial mundial; pese a que en años anteriores fue un medio de transporte que perdió fuerza en el comercio, en los últimos 20 años ha ido recobrando terreno, en gran medida debido a los grandes avances tecnológicos presentes tanto en los puertos como en la nueva generación de buques y en general de todo el sistema logístico que involucra (UNCTAD, 2017).

Numerosos estudios afirman la importancia de la conectividad y el desempeño logístico como factores decisivos en la competitividad del comercio exterior, al coadyuvar a disminuir los costos del comercio internacional (Fugazza y Hoffman, 2017; Wilmsmeier et al., 2017 y Hoffmann et al., 2017). En el caso de los barcos, cabe señalar que se han ido adaptando a las demandas del mercado además de sus grandes ventajas como son el bajo costo y la gran capacidad de los barcos de carga. De forma tal que, ha resultado ser un medio de transporte bastante eficiente que permite conectar puntos muy lejanos a bajos costos respecto a su capacidad de carga tanto en volumen como en tonelaje. Actualmente existen diferentes tipos de embarcaciones de carga que van desde barcos especializados hasta los grandes buques de contenedores con mercancías prácticamente de todo tipo.

Por su parte, los puertos han ido también teniendo un papel muy importante en el comercio exterior y gracias al uso de la tecnología e infraestructura de vanguardia en las instalaciones portuarias, ahora es posible una mayor conectividad entre el medio marítimo y terrestre lo que permite un flujo creciente en el transporte intermodal (SCT, 2013). Para fines logísticos, se puede dividir a los puertos en tres tipos, dependiendo de la zona en la que se encuentren: la zona marítima o de acceso, la terrestre para maniobras y la de enlace con los modos terrestres (carretero y férreo).

La zona marítima como su nombre lo indica permite la entrada de las diferentes embarcaciones que arriban al puerto, a través de un canal principal de acceso, la dársena ${ }^{1}$ de la ciaboga ${ }^{2}$ y los canales secundarios hacia sus distintas posiciones de atraque. Su capacidad está limitada, por una parte, al ancho de los canales y al diámetro de la dársena, así como a la profundidad mínima de sus aguas, que es conocida como el calado. Siendo esta una de las ventajas competitivas del puerto de Lázaro Cárdenas, ya que sus áreas de navegación de hasta $18 \mathrm{ml}$. (59 ft) para embarcaciones de gran calado y capacidad de desplazamiento de 170,000 t., le dan gran ventaja sobre otros puertos. (SCT, 2017).

\footnotetext{
${ }^{1}$ Es la parte resguardada artificialmente, en aguas navegables, para el surgidero o para la carga y descarga cómoda de embarcaciones.

${ }^{2}$ Es la maniobra que tiene por objeto hacer que un barco gire o vire en redondo en el menor espacio posible.
} 
La zona terrestre, está integrada principalmente por los muelles ubicados en las diferentes terminales con las que cuenta cada puerto comercial, con base en la vocación de los distintos tipos de mercancías (gráneles, líquidos, vehículos, carga general, refrigerada y contenedores, entre otras) y el equipamiento de que disponen para efectuar las maniobras de carga y descarga de los buques.

Actualmente, la zona terrestre del puerto de Lázaro Cárdenas, se compone de un punto de inspección fito-zoosanitaria y doce terminales: terminal de granos, dos terminales de minerales a granel y productos de acero, tres terminales de usos múltiples, dos terminales de contenedores, terminal de metales y minerales, terminal de fluidos petroleros, terminal de carbón, terminal de fertilizantes y terminal de desmantelamiento de embarcaciones y manejo de fluidos (APM, 2018).

En lo que corresponde a la zona de enlace, en ella se encuentran las superficies e instalaciones que permiten el acceso, circulación, estacionamiento y operación de los modos de transporte terrestre de carga, así como las destinadas al almacenamiento de transferencia de las mercancías operadas, tanto de importación como de exportación, como son los circuitos de reconocimiento aduanero, bodegas fiscalizadas y las oficinas de las distintas autoridades, servicios y actores privados que actúan dentro del recinto portuario.

A su vez, los puertos se dividen en dos categorías acorde al tipo de tráfico que reciben: los de altura, que reciben flujos internacionales y, los de cabotaje que únicamente reciben flujos nacionales. México dispone de $11,500 \mathrm{~km}$ de litorales, de alrededor de 117 instalaciones portuarias de distintos tipos y vocaciones (ver figura 1), entre las que se cuentan puertos de abrigo, comerciales, industriales, petroleros, pesqueros, turísticos y para fines militares y de seguridad nacional (SCT, 2013).

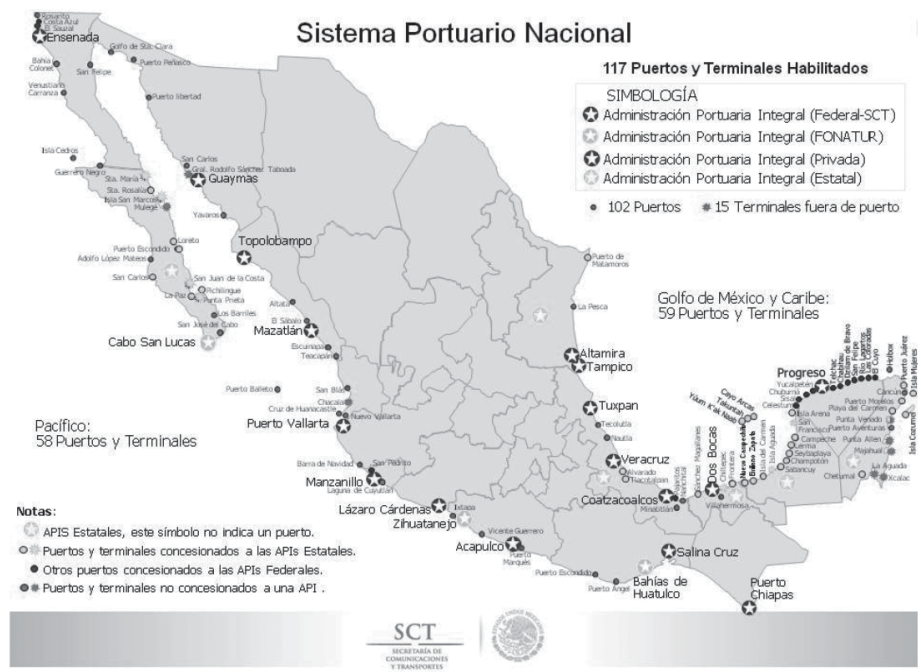

Figura 1. Sistema Portuario Nacional Fuente: SCT, 2012. 
Actualmente, en materia de instalaciones portuarias comerciales, las de mayor relevancia en el país, son las de los puertos de Manzanillo y Lázaro Cárdenas en el litoral del Pacífico, y los de Veracruz y Altamira en el litoral del Golfo de México.

El puerto Lázaro Cárdenas cuenta con 18.00 metros de profundidad en su canal de acceso y 16.50 metros de profundidad en la dársena principal de ciaboga. Además, se encuentra protegido para recibir embarcaciones de hasta 165 mil toneladas de desplazamiento. Lo que los hace como ya se mencionó anteriormente, un puerto único en su clase por su gran capacidad para recibir buques de gran calado.

Otra de las características que permiten hablar de conectividad y competitividad en los servicios logísticos es el factor tiempo, por lo que resulta importante destacar la distancia del puerto de Lázaro Cárdenas tanto al interior de la República Mexicana como a los diferentes puertos internacionales con los que se comunica. Como se observa en la tabla 1 el puerto de Lázaro Cárdenas es un punto estratégico entre diferentes puntos de la república donde destaca particularmente la conexión con la Ciudad de México, Monterrey, San Luis Potosí y Altamira.

Tabla 1

Distancia del Puerto de Lázaro Cárdenas al interior de la República Mexicana

\begin{tabular}{|c|c|c|c|c|c|}
\hline Ciudad & $\begin{array}{l}\text { Punto Intermodal } \\
\text { y Multimodal }\end{array}$ & $\begin{array}{l}\text { Distancia } \\
(\mathrm{km})\end{array}$ & $\begin{array}{l}\text { Tiempo } \\
\text { aprox. (horas.) }\end{array}$ & Ferrocarril & $\begin{array}{l}\text { Posibilidad para } \\
\text { desaduanar }\end{array}$ \\
\hline Ciudad de México & $\begin{array}{l}\text { Terminal Intermodal Pantaco } \\
\text { (Ferrovalle) }\end{array}$ & 863 & 42 & KSC & SI \\
\hline Cuautitlán, Edo. Mex. & $\begin{array}{l}\text { Terminal Contrimodal, S.A. } \\
\text { de C.V. }\end{array}$ & 863 & 42 & $\mathrm{KSC}$ & SI \\
\hline Monterrey & $\begin{array}{l}\text { Terminal Intermodal KSC de } \\
\text { México }\end{array}$ & 1292 & 64 & $\mathrm{KSC}$ & SI \\
\hline Guadalajara & $\begin{array}{l}\text { Terminal de carga intermodal de } \\
\text { Guadalajara (TCIG) }\end{array}$ & 896 & 66 & $\begin{array}{l}\text { KSC- } \\
\text { Ferromex }\end{array}$ & SI \\
\hline Querétaro & $\begin{array}{l}\text { Servicios Integrales y Desarro- } \\
\text { llo GMG (SID) }\end{array}$ & 617 & 40 & $\mathrm{KSC}$ & SI \\
\hline Veracruz & $\begin{array}{l}\text { Internacional de Contenedores } \\
\text { Asociados de Veracruz. S.A. }\end{array}$ & 1303 & 64 & $\mathrm{KSC}$ & SI \\
\hline San Luis Potosí & $\begin{array}{l}\text { Terminal Intermodal de carga } \\
\text { KSC de México }\end{array}$ & 792 & 44 & $\mathrm{KSC}$ & SI \\
\hline Altamira & $\begin{array}{l}\text { Altamira Terminal Intermodal, } \\
\text { S.A. de C.V. }\end{array}$ & 1266 & 64 & $\begin{array}{l}\text { KSC- } \\
\text { Ferromex }\end{array}$ & SI \\
\hline Toluca & $\begin{array}{l}\text { Terminal Maclovio Herrera } \\
\text { (KSC de México) }\end{array}$ & 897 & 48 & $\mathrm{KSC}$ & $\mathrm{NO}$ \\
\hline Laredo & $\begin{array}{l}\text { Terminal de KSC de México, } \\
\text { Laredo }\end{array}$ & 1558 & 94 & $\mathrm{KSC}$ & SI \\
\hline
\end{tabular}

Fuente: SCT, 2016.

Respecto a la conectividad con otros países, cabe señalar que dentro del puerto operan distintas navieras destacando entre ellas Maersk Line, APL, HAPAG-LLOYD, que conectan al puerto con Norte América, Centro América, América del Sur y Asia.

\section{Metodología}

Las redes neuronales artificiales (RNA) son una representación matemática, inspirada en el cerebro humano, tomando como base el sistema de procesamiento de la información, así como, la estructura sistémica que sigue el flujo de la información. Se puede afirmar, desde un punto de 
vista matemático que es una herramienta no linear para optimizar la información. Acorde con Freman y Skapura (1993), es un sistema de procesadores paralelos conectados entre sí en forma de grafo dirigido. Esquemáticamente cada elemento de procesamiento (neuronas) de la red se representa como un nodo. El desarrollo de la técnica de las RNA permite aprender, reconocer y aplicar relaciones entre objetos y tramas de objetos propios del mundo real (Sotolongo y Guzmán, 2001).

La técnica de RNA de modo simple consiste en sumar los valores de las entradas (inputs) que recibe de otras unidades conectadas a ella, los valores se comparan con el valor umbral $\mathrm{y}$, si lo iguala o supera, envía una activación o salida (output) a las unidades a las que esté conectada. Tanto las entradas que la unidad recibe como las salidas que envía dependen a su vez del peso o fuerza de las conexiones por las cuales se realizan dichas operaciones, por lo que adicionalmente la técnica permite ver el peso, fuerza de conexión o grado de importancia entre las variables analizadas y la variable dependiente (Figueredo y Ballesteros, 2016).

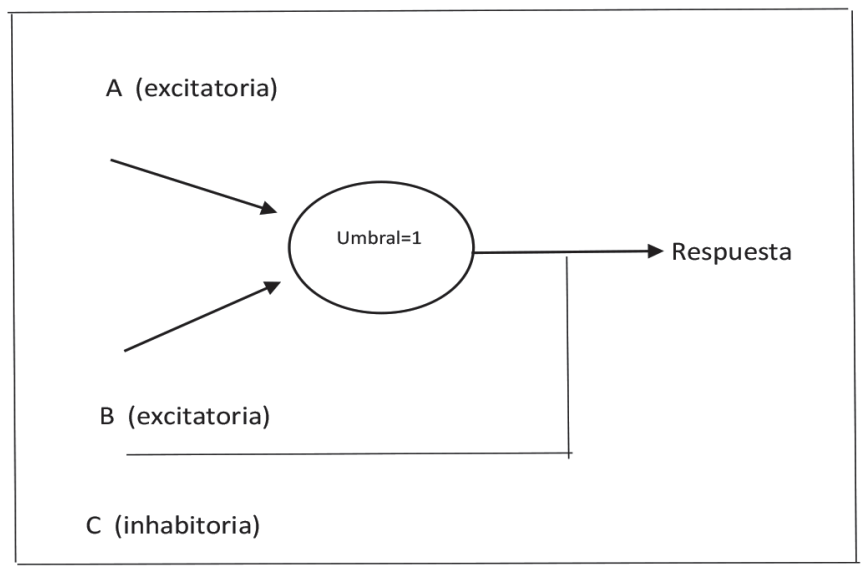

Figura 2. Neurona de McCulloch-Pitts. Fuente: Montaño (2002).

La diferencia de las redes neuronales respecto de otros métodos estadísticos tradicionales como puede ser una regresión lineal, es que mientras la regresión lineal parte de una estructura de datos de modelo rígida y un conjunto de supuestos que es preciso imponer antes del modelaje de los datos, las RNA forman relaciones entre las variables durante el procesamiento de los datos de tal forma que, si la relación variable dependiente y variable(s) independiente(s) es adecuada la RNA mostrara un resultado acorde a la regresión lineal, pero en caso de que no sea así, la RNA mostrará la asociación o estructura de modelo "correcta".

Entre las ventajas de la aplicación de la RNA destacan: la capacidad de aprender a realizar tareas basadas en una experiencia inicial y el entrenamiento; la fácil inserción dentro de la tecnología existente; la capacidad de realizar operaciones en paralelo; y la auto-organización de la red a través de procesos de aprendizaje.

Otra característica importante de esta técnica es, que no presupone una pre-programación del sistema, de ahí que el comportamiento del sistema es libre para que las variables interactúen 
acorde a sus propias conexiones; así mismo la técnica no sigue un procesamiento secuencial, ya que el procesamiento se realiza en paralelo (Montaño, 2002).

\section{Estructura de una RNA}

Las redes neuronales artificiales, están formadas por una gran cantidad de neuronas, estas no suelen denominarse neuronas artificiales sino nodos o unidades de salida. Un nodo o neurona cuenta con una cantidad variable de entradas que provienen del exterior (X1, X2, ....., Xm). A su vez, dispone de una sola salida $(\mathrm{Xj})$ que transmitirá la información al exterior o hacia otras neuronas. Cada Xj o señal de salida tiene asociada una magnitud llamada peso este se calculará en función de las entradas, por lo cual cada una de ellas es afectada por un determinado peso $(\mathrm{Wjo...Wjq}+\mathrm{m})$. El proceso que sigue el modelo, consiste en ir encontrando el peso de cada una de las variables y las interrelaciones que se generan y que codifican los conocimientos. Al conectar varias neuronas de un determinado modo, se obtiene una red. Existen variaciones de topología, se clasifican según tres criterios (Sotolongo y Suárez, 2000):

1. Número de niveles o capas.

2. Número de neuronas por nivel.

3. Formas de conexión.

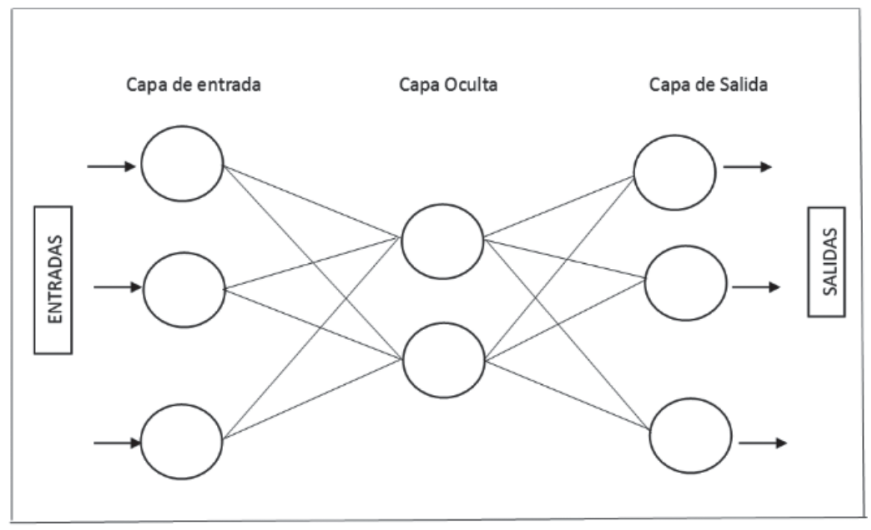

Figura 3. Arquitectura de un Perceptrón multicapa. Fuente: Montaño (2002).

Existen varias técnicas dentro del modelaje de RNA, como son la de perceptrón multicapa y la función de base radial. El procesamiento perceptrón multicapa (MLP) así como la función de base radial (FBR), generan un modelo predictivo para una o más variables dependientes o destino con base en los valores de las variables predictivas. No obstante, los MLC permiten relaciones más complejas con el coste posible de aumentar el tiempo de entrenamiento y puntuación. La FBR puede tener tiempos de entrenamiento y puntuación inferiores, con el coste posible de una potencia de predicción reducida en comparación con MLC, por lo que la selección entre un tipo de modelo y otro, radica en gran medida en el volumen de la información, así como las variables del modelo. 
El procesamiento perceptrón multicapa (MLP) también conocido como el algoritmo de backpropagation es una extensión de la regla propuesta por Widrow y Hoff en 1960 (regla delta) que tiene como objetivo propagar los errores cometidos por las unidades de salida hacia atrás, ya que, en un sistema de este tipo, el error cometido por una unidad intermedia depende del error cometido por las unidades de salida a las que dicha unidad intermedia está conectada (Rodríguez y Turias, 2016). Tras conocerse el error cometido por las unidades intermedias, pueden entonces modificarse las conexiones entre unidades de entrada y unidades intermedias. De forma similar a la regla delta, la base matemática del algoritmo backpropagation es la técnica de gradiente decreciente, basada en modificar los pesos en la dirección opuesta al gradient $^{3}$, esto es $-\partial \mathrm{Ep} / \partial \mathrm{w} \mathrm{ij}$, en la dirección que determina el decremento más rápido del error (Montaño, 2002).

\section{Selección de variables}

De acuerdo con el Banco Mundial (2014) y con la Organización Mundial de Comercio (2015) pueden identificarse 5 factores que son críticamente importantes para las cadenas de producción, dichos factores existen en la planificación de todas las cadenas de producción, pero son relativamente más dominantes en términos de combinaciones y extensión en un contexto global. Dichos factores son los siguientes: almacenaje, transporte, administración aduanera, costos y tiempo. A continuación, se describen cada uno de estos factores, que para el caso de la presente investigación fungirán como variables:

a.Administración Aduanera. Las aduanas son organismos gubernamentales localizados estratégicamente en los puntos de entrada y salida de un país. Sus funciones principales son permitir el flujo de mercancías atendiendo a las regulaciones fiscales correspondientes así como salvaguardar la seguridad nacional. La existencia de una buena administración en las aduanas, garantiza que existan controles eficaces que aseguren la recaudación de ingresos, el cumplimiento de la legislación y la seguridad nacional así como la circulación de las mercancías de manera fluida. La eficacia y eficiencia de los procedimientos aduaneros tienen una influencia considerable en la competitividad económica de los países, el crecimiento del comercio internacional y por ende den el desarrollo del mercado mundial.

b. Almacenaje. Los almacenes o Warehounsing son espacios cercanos a las aduanas, que tienen la función de custodiar y la capacidad de almacenar y manipular mercancías, optimizando con ello el traslado de la misma a los puntos de estiba o transportación.

c. Tiempos. A pesar de las distancias y la incertidumbre de algunos fenómenos susceptibles a suceder en el traslado de las mercancías de un lugar a otro, hoy en día ante la gran competencia entre productos y la exigencia creciente de los consumidores, el factor tiempo desempeña un papel fundamental ya que, la entrega puntual en un lugar y tiempo determinado, puede hacer la diferencia entre un producto considerado competitivo internacionalmente y la perdida de esa competitividad.

d. Transporte. Este factor se refiere al traslado de la mercancía de un punto a otro; los tipos de transporte acorde con la ley aduanera mexicana son: marítimo y fluvial, férreo, carretero, aéreo, cables y ductos. Sin embargo, en materia de comercio exterior,

\footnotetext{
${ }^{3}$ Variación de una magnitud en función de la distancia, a partir de la línea en que esta variación es máxima en las magnitudes cuyo valor es distinto en los diversos puntos de una región del espacio.
} 
también se utiliza el término de transporte multimodal, es decir el uso de diferentes tipos de transporte dentro del sistema logístico, a fin de llevar la mercancía hasta el punto acordado con el cliente de manera más eficiente, reduciendo así tiempos y costos.

e. Costos. En cualquier sistema de comercio, el costo tiene a ser un factor clave en la determinación de la competitividad, por lo que los montos monetarios adicionales a la elaboración del producto, relacionados con los servicios logísticos no deben ser altos, puesto que estos impactarán en el precio final del producto.

Una vez explicadas las cinco principales variables, se precedió a la recolección de la información de estas. La recolección se hizo de fuentes directas de información, por medio de entrevistas y cuestionarios estructurados, aplicados a 68 empresas especializadas en el área, las cuales, según la compañía, tipo de empresa, área de desarrollo y función, brindaron información necesaria para tener una visión amplia respecto de las variables analizadas. El cuadro 1, sintetiza la información básica recogida de las encuestas aplicadas a los especialistas de dichas empresas. Cabe señalar que, las variables de entrada a la red se determinaron por medio de un modelo autorregresivo.

Tabla 2

Expertos en el campo laboral del comercio internacional entrevistados

\begin{tabular}{|c|c|c|c|c|}
\hline Compañía & Tipo de empresa & Área & Número de encuestados & Función \\
\hline LC Logistics GPS & Recinto fiscalizado & Comercial & 10 & Búsqueda de clientes \\
\hline LCTPC & Recinto fiscalizado & Comercial & 16 & $\begin{array}{l}\text { potenciales, ventas, } \\
\text { atención al cliente }\end{array}$ \\
\hline Mitsui Co. & $\begin{array}{l}\text { Empresa distribui- } \\
\text { dora de láminas } \\
\text { de acero }\end{array}$ & Tráfico y logística & 3 & $\begin{array}{l}\text { Búsqueda de clientes } \\
\text { potenciales, ventas, } \\
\text { atención al cliente }\end{array}$ \\
\hline $\begin{array}{l}\text { Steel Technologies de } \\
\text { México }\end{array}$ & $\begin{array}{l}\text { Fabricación y venta } \\
\text { de placas de acero }\end{array}$ & Tráfico y logística & 2 & $\begin{array}{l}\text { Coordinador de logística } \\
\text { Coordinador de logística }\end{array}$ \\
\hline Hanwa Steel Service & $\begin{array}{l}\text { Empresa distribui- } \\
\text { dora de láminas } \\
\text { de acero }\end{array}$ & Tráfico y logística & 2 & Coordinador de logística \\
\hline Eurotranciatura & $\begin{array}{l}\text { Productora de } \\
\text { láminas eléctricas } \\
\text { de acero }\end{array}$ & Tráfico y logística & 2 & Coordinador de logística \\
\hline Nippon Express & $\begin{array}{l}\text { Compañía especia- } \\
\text { lizada en Logística } \\
\text { global }\end{array}$ & Tráfico y logística & 2 & Coordinador de logística \\
\hline V Modal Mexicana & $\begin{array}{r}\text { Servicios de Logís } \\
\text { Ferroviario I }\end{array}$ & $\begin{array}{l}\text { tica y Transporte } \\
\text { ntermodal }\end{array}$ & 3 & Tráfico y Aduanas \\
\hline $\begin{array}{l}\text { Servilamina Summit } \\
\text { Mexicana }\end{array}$ & $\begin{array}{l}\text { Distribución de } \\
\text { láminas de acero }\end{array}$ & Logística y tráfico & 2 & Coordinador de logística \\
\hline Polímeros Mexicanos & $\begin{array}{l}\text { Exportadora de } \\
\text { polímeros }\end{array}$ & $\begin{array}{l}\text { Jefe de exporta- } \\
\text { ciones }\end{array}$ & 3 & $\begin{array}{l}\text { Logística de exporta- } \\
\text { ción, trámites necesarios }\end{array}$ \\
\hline Nicometal Mexicana & $\begin{array}{l}\text { Productora de } \\
\text { láminas de acero }\end{array}$ & Tráfico y logística & 3 & Coordinador de logística \\
\hline Ulises Anaximandro & $\begin{array}{l}\text { Importadora de } \\
\text { Triplay }\end{array}$ & Dirección general & 1 & $\begin{array}{l}\text { Supervisar y aprobar } \\
\text { todos los procesos } \\
\text { dentro de la empresa }\end{array}$ \\
\hline Nicometal Hidalgo & $\begin{array}{l}\text { Productora de } \\
\text { láminas de acero }\end{array}$ & Tráfico y logística & 3 & Coordinador de logística \\
\hline Oñate Willy y Cía. & Agencia Aduanal & Jefe de tráfico & 2 & $\begin{array}{l}\text { Garantizar que todos } \\
\text { los despachos salgan en } \\
\text { tiempo y forma }\end{array}$ \\
\hline
\end{tabular}




\begin{tabular}{|c|c|c|c|c|}
\hline Visa Logística & Agencia Aduanal & Despacho & 1 & $\begin{array}{l}\text { Cumplir con los trámites } \\
\text { necesarios para el des- } \\
\text { pacho de mercancías }\end{array}$ \\
\hline $\begin{array}{l}\text { Agencias Aduanales } \\
\text { Padilla }\end{array}$ & Agencia Aduanal & Ejecutivo de tráfico & 2 & $\begin{array}{l}\text { Gestionar todos los trá- } \\
\text { mites para el despacho } \\
\text { de mercancías }\end{array}$ \\
\hline $\begin{array}{l}\text { Agencia Aduanal } \\
\text { CONIA }\end{array}$ & Agencia Aduanal & Ejecutivo de tráfico & 2 & $\begin{array}{l}\text { Gestionar todos los trá- } \\
\text { mites para el despacho } \\
\text { de mercancías }\end{array}$ \\
\hline $\begin{array}{l}\text { Muris Salinas } \\
\text { Consultores }\end{array}$ & Agencia Aduanal & $\begin{array}{l}\text { Ejecutivo de } \\
\text { exportaciones }\end{array}$ & 3 & $\begin{array}{l}\text { Gestionar todos los } \\
\text { trámites para la exporta- } \\
\text { ción de mercancías }\end{array}$ \\
\hline Valdez \& Woodward & Agencia Aduanal & $\begin{array}{l}\text { Jefe de operaciones } \\
\text { / Tráfico }\end{array}$ & 2 & $\begin{array}{l}\text { Revisión y cumpli- } \\
\text { mientos de las normas y } \\
\text { procesos establecidos }\end{array}$ \\
\hline $\begin{array}{l}\text { Logística Corporativa } \\
\text { en Comercio Exterior }\end{array}$ & Agencia Aduanal & Ejecutivo de tráfico & 2 & $\begin{array}{l}\text { Gestionar todos los trá- } \\
\text { mites para el despacho } \\
\text { de mercancías }\end{array}$ \\
\hline Luis Hoyo & Agencia Aduanal & Tráfico & 2 & $\begin{array}{l}\text { Gestionar todos los trá- } \\
\text { mites para el despacho } \\
\text { de mercancías }\end{array}$ \\
\hline
\end{tabular}

Fuente: Elaboración propia con base en las entrevistas y encuestas aplicadas a las empresas .También con datos INEGI.

\section{Resultados}

Los resultados obtenidos a través del método de RNA se dividieron en dos partes a fin de tener una visión más clara de los mismos. En la primera parte, se analizó el pronóstico de satisfacción por variable considerando cinco posibles respuestas, dicho pronóstico se presenta en la curva de COR auxiliada de los porcentajes de área debajo de la curva obtenidos. En la segunda parte, se presenta el análisis de importancia acorde a las redes de cada una de las variables independientes respecto de la variable dependiente analizada, para lo cual se analizaron las cinco fases del sistema logístico antes mencionadas; cabe agregar que, se consideró a cada una, como variable independiente respecto de los indicadores de las mismas.

Para todas las variables analizadas, la arquitectura de la red fue de tipo feedforward, en otras palabras, las conexiones de la red fluyeron de manera unidireccional desde la capa de entrada a la capa de salida, sin ciclos de retroalimentación, en una estructura de tres capas: de entrada, oculta y de salida. Cabe recordar que los valores de la capa de entrada, fueron de tipo ordinal, debido a que se extraen de un cuestionario donde los valores de respuesta representan cinco categorías para cada pregunta. A fin de obtener mejores resultados se corrieron los datos en dos conjuntos de datos activos correspondientes a la muestra de entrenamiento y la muestra de prueba.

La muestra de entrenamiento comprende los registros de datos utilizados para entrenar a la red neuronal, introduciéndole cierto porcentaje de casos del conjunto de datos que debe asignarse a la muestra de entrenamiento, para poder obtener un modelo. La muestra de prueba, es un conjunto independiente de registros de datos que se utiliza para realizar un seguimiento de los errores durante el entrenamiento ambas pruebas se pueden ver en el anexo.

Respecto a las capas ocultas, cada modelo mostró solo una capa cuya función de activación fue del tipo tangente hiperbólica, $\gamma(c)=\tanh (c)=\left(e^{c}-e^{-c}\right) /\left(e^{c}+e^{-c}\right)$ que permite transformar los 
argumentos en rangos que oscilan en valores numéricos de entre -1 a +1 ; finalmente para la capa de salida, se utilizó la función de activación de identidad, dicha función tiene la siguiente forma: $\gamma(c)=c$. De dichos análisis se desprende un análisis de susceptibilidad, que calcula la importancia de cada predictor en la determinación de la red neuronal. El análisis se basa en las muestras de entrenamiento y comprobación combinadas. Así, se crea una tabla y un gráfico que muestra la importancia normalizada de cada predictor.

\section{Pronósticos de satisfacción por variable}

La curva COR (Característica Operativa del Receptor; ROC, en inglés) proporciona una representación visual de la susceptibilidad y especificidad para todos los cortes posibles en un único gráfico, que es mucho más limpio y potente que una serie de tablas. El gráfico es la representación de la razón o ratio de verdaderos positivos (VPR = Razón de Verdaderos Positivos) frente a la razón o ratio de falsos positivos (FPR = Razón de Falsos Positivos). Así como también representa el umbral de discriminación (valor a partir del cual se decide si un caso es positivo) por lo que el gráfico, muestra diferentes categorías con respecto a una línea de 45 grados, que va de la esquina superior izquierda del gráfico a la esquina inferior derecha (Fogarty, Baker \& Hudson, 2005).

Cabe señalar que, el gráfico se basa en las muestras de prueba y entrenamiento combinadas. Por otra parte, el área bajo la curva es un resumen numérico de la curva COR, y los valores de la tabla representan, para cada categoría, la probabilidad de que la pseudoprobabilidad pronosticada de estar en esa categoría, sea mayor para un caso elegido aleatoriamente en esa categoría, que para un caso elegido aleatoriamente que no pertenezca a esa categoría.

Como se puede observar, los valores pronosticados para las variables aduana, almacenaje y tiempo se presentan en la figura 4. Las categorías para el pronóstico son cinco para todos los casos, no obstante, algunas categorías no tuvieron un valor arriba de cero por lo que no están representadas en el gráfico, lo que significa que su valor pronosticado de que suceda es cero.

En el caso de la variable aduana, se observa que la probabilidad pronosticada respecto a la calidad del servicio acorde con la curva COR y el área bajo la curva, varía desde "muy mala" (0.936) a "ni bueno ni malo" (0.784) lo que permite vislumbrar un bajo grado de satisfacción del servicio proporcionado por las aduanas.

Por su parte, el servicio de almacenaje es considerado como algo mayoritariamente necesario, ya que los rangos más altos encontrados en el pronóstico acorde con el área bajo la curva van desde "muy necesarias" (0.862) a "ni necesarias ni innecesarias" con (0.915). Por su parte la variable tiempo, acorde con el modelo, es percibida como una variable fundamental ya que el valor pronosticado para "algo en desacuerdo" y "nada de acuerdo" es cero además de que los valores más altos se encuentran dentro de las opciones "algo de acuerdo" (0.887) y "muy de acuerdo" (0.777) (ver figura 4). 


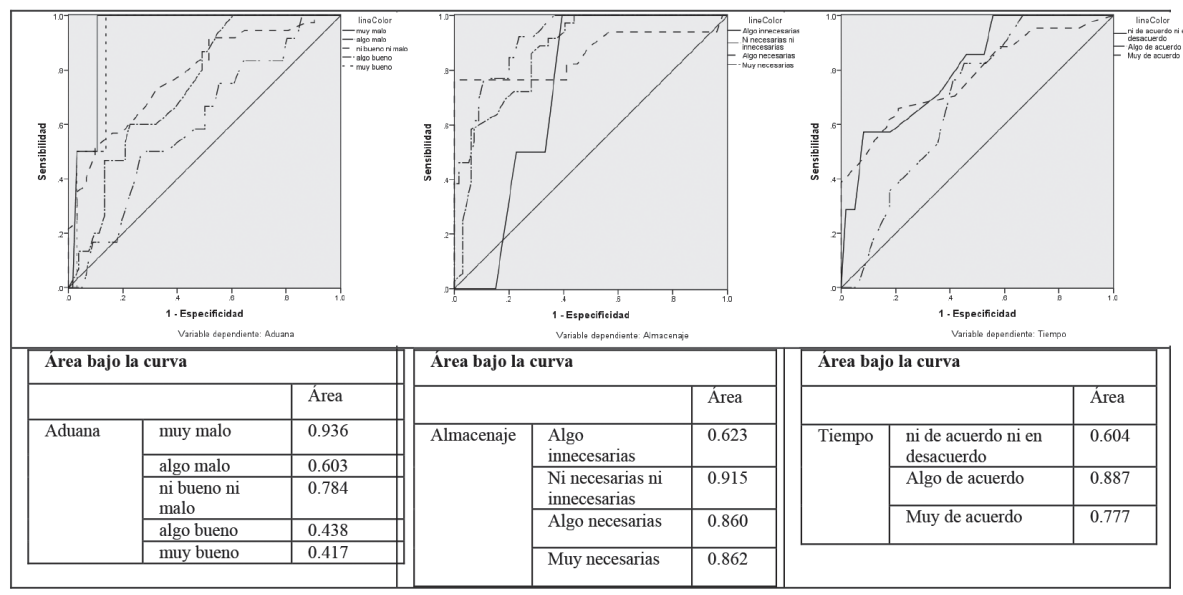

Figura 4. Pronóstico de satisfacción por variable para las variables Aduana, Almacenaje y Tiempo, curva COR. Fuente: Elaboración propia con base en redes neuronales, perceptrón multicapa.

Los resultados respecto a la satisfacción de la variable transporte, reflejan una satisfacción media a superior respecto del servicio, ya que el área bajo la curva va de las opciones "ni de acuerdo ni en desacuerdo" (0.665) a "muy de acuerdo" (0.502). Finalmente, el valor pronosticado para el costo de los servicios logísticos es un tanto variable, ya que casi todas las opciones oscilan en valores de (0.604 a 0.799) exceptuando la opción "muy barato" (véase figura 5).

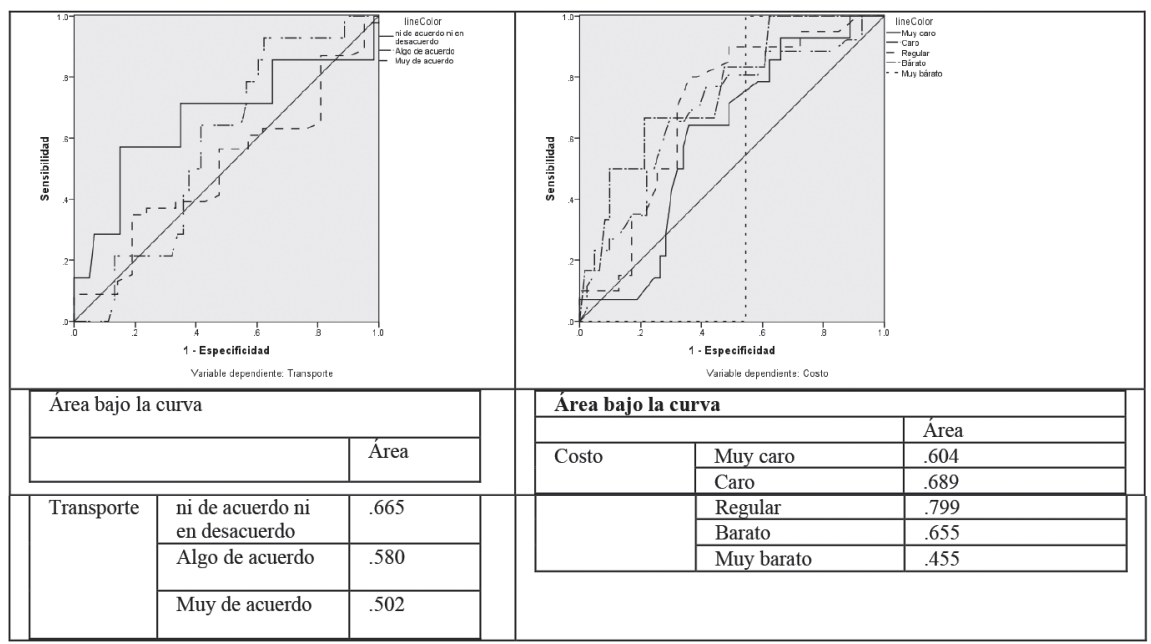

Figura 5. Pronóstico de satisfacción por variable para las variables Transporte y Costo, curva COR. Fuente: Elaboración propia con base en redes neuronales, perceptrón multicapa. 


\section{Importancia de las variables independientes}

Una vez analizadas las variables dependientes desde el punto de vista de su importancia y grado de satisfacción pronosticado, se presentan los resultados para cada una las variables (almacenaje, transporte, administración aduanera, costos y tiempo) respecto de sus variables independientes, con la finalidad de determinar que variables independientes son más importantes, respecto de la variable dependiente analizada.

Las variables independientes, para cada variable dependiente analizada y que pasaron la prueba de correlación de Pearson, son las variables que se presentan en la tabla 2. A pesar de que se sabe que existe una correlación entre las partes, no se conoce cuál es grado de importancia de la misma por lo que la tabla 2 muestra el grado de importancia de cada variable, después de un proceso de normalización de los datos para mejorar la comprensión y análisis de los resultados.

Para la metodología de RNA, la importancia de una variable independiente es una medida que indica cuánto cambia el valor pronosticado (por el modelo de la red) para diferentes valores de la variable independiente. Mientras que, la importancia normalizada es el resultado de los valores de importancia divididos por los valores de importancia mayores expresados como porcentajes.

Las variables independientes, respecto de la variable dependiente aduana, son tres: apoyos gubernamentales, regulaciones y tiempo de despacho aduanero. Donde las más importantes, acorde a la metodología empleada son: apoyos gubernamentales, con un 100 por ciento de importancia, seguida del tiempo de despacho aduanero (89.7 por ciento) y el número de regulaciones al comercio exterior, con un 46.3 por ciento de importancia.

Es claro que, desde la perspectiva de los especialistas y los teóricos en economía internacional, el gobierno juega un papel fundamental en el comercio exterior. La reducción de aranceles y otros instrumentos comerciales que se han venido dando en los últimos años, así como la facilitación de trámites en la administración aduanera, inciden directamente en la percepción eficiente del trabajo de las aduanas. Por otra parte, el factor tiempo, en el proceso de desaduanamiento, -particularmente cuando el sistema aleatorio que forma parte del despacho aduanero obliga a una inspección- es vital para la eficiencia del sistema logístico de cualquier producto que busque competir en el comercio exterior, de ahí que diferentes países, incluyendo México, han orientado a sus aduanas a sistemas electrónicos buscando agilizar y digitalizar trámites.

Respecto a la variable regulaciones, a pesar de ser una variable importante su importancia resulto menor en el modelo, esto se puede explicar por la exigencia cada vez mayor del consumidor que obliga a las empresas a mejorar sus estándares de calidad, cumpliendo de antemano muchas de las regulaciones revisadas en el paso por la aduana.

Para el caso de la variable dependiente almacenaje, la variable más importante es el manejo de la mercancía, con un 100 por ciento de importancia, lo que resulta coherente puesto que, un buen manejo de la mercancía garantiza la llegada de la misma en las mejores condiciones posibles. Es importante señalar que, el manejo de mercancía en almacén abarca actividades como: traslados dentro del almacén, carga y descarga, un sistema de control de inventarios, control de plagas y condiciones ambientales adecuadas entre otras actividades.

La variable independiente que sigue en importancia, es el equipo, con un 56.7 por ciento esto quiere decir que el almacén debe contar con el equipo necesario para llevar a cabo las transacciones en comercio exterior; como suelen ser el equipo de distribución para mover las mercancías y entregar los productos, equipos de empaque y embalaje, entre otros. La variable 
ubicación recibió un 34.4 por ciento de importancia mostrando que, efectivamente es algo a considerar, pero no tan significante como los aspectos anteriormente mencionados, lo cual implica que un almacén que cuente con un excelente manejo y el equipo necesario pese a estar lejos, serán preferidos a la hora de seleccionar el servicio logístico a contratar.

Por último, la variable especialización de los almacenes recibió un 28.5 por ciento de importancia, lo cual implica que, gran parte de los usuarios no tienen la necesidad de servicios especializados para productos muy específicos o particularmente tecnológicos. Esto, se puede explicar porque el puerto de Lázaro Cárdenas recibe mercancía muy variada, caso contrario a algunas aduanas con comercio exterior especializado.

Respecto a la variable tiempo, son dos las variables independientes consideradas: duración del traslado y cumplimiento con el tiempo estipulado. Efectivamente, la duración del traslado puede ser una variable de gran significancia dependiendo del tipo de producto o mercancía que se esté transportando. Un claro ejemplo de esto, puede ser la urgencia de trasladar productos perecederos antes de que comience el proceso de descomposión de estos. No obstante, el grado de importancia percibido fue de un 27. 2 por ciento acorde a los resultados obtenidos. Este resultado, se explica en gran medida por la especificidad de los productos en cuestión como ya se mencionó. Mientras que, la variable cumplimiento de los plazos establecidos, obtuvo un grado de importancia del 100 por ciento, reflejando con ello que es un factor clave en la competitividad de los servicios logísticos, cumplir con los tiempos de servicio conforme a lo previamente pactado.

Las variables independientes consideradas respecto de la variable transporte, fueron: número de unidades, rutas fiscales, consolidación de envíos, conocimiento respecto al trasporte seleccionado y tipos de transporte a disposición.

Exceptuando la variable rutas fiscales, que mostró un 100 por ciento de importancia, todas las variables seleccionadas mostraron un nivel de importancia media, con valores que oscilan entre el 50 por ciento (número de unidades) al 41.3 por ciento (tipos de transporte) mostrando un rango relativamente importante, pero al mismo tiempo uniforme. En cuanto a la variable rutas fiscales considerada acorde a los resultados como la variable más importante de este rubro, denota la necesidad de alternativas que conecten al puerto de Lázaro Cárdenas con el resto del mundo, no solo mediante la vía marítima sino también con la intensificación de las rutas férreas, las rutas fiscales carreteras y conexiones aéreas.

Es importante destacar que, para la variable costos de los servicios logísticos, se consideraron las variables independientes, en orden de importancia: tipos de servicios logísticos que obtuvo un valor de importancia del 100 por ciento, lo que reitera que el costo debe estar íntimamente relacionado con los servicios que se ofrecen; calidad de los servicios, con un 96.3 por ciento de importancia, lo que implica que los usuarios de los servicios, están dispuestos a pagar un costo más alto siempre y cuando los estándares de los servicios sean también altos.

Por su parte, la relación costo volumen en importación y exportación, mostró un grado de importancia de 46.2 por ciento, lo que indica que efectivamente es importante la relación volumen costo, pero no determinante, cabe señalar que, dependiendo de la mercancía a comercializar, el costo puede ser determinado por el peso o incluso valor de la mercancía en lugar del volumen. 

http://dx.doi.org/10.22201/fca.24488410e.2018.1494

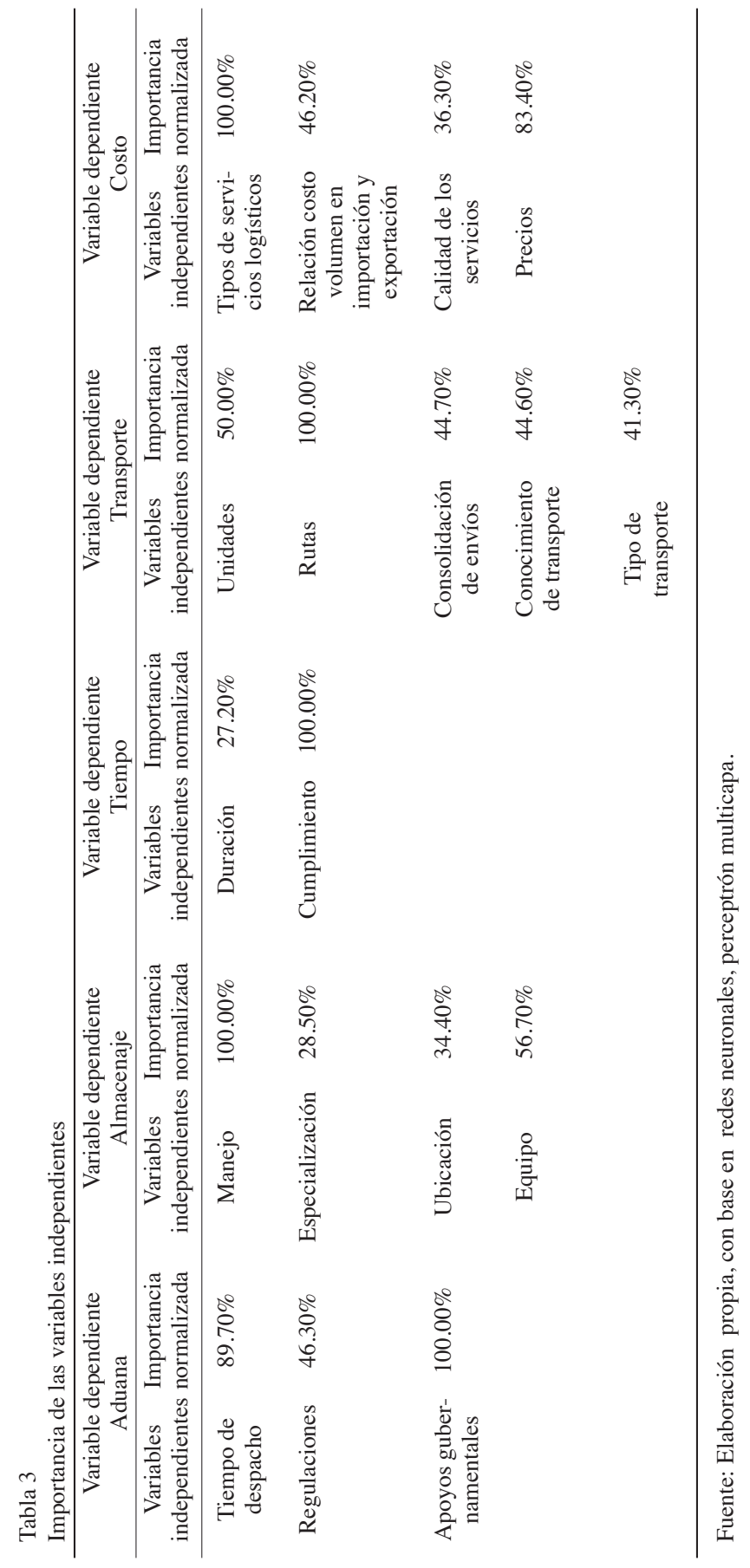




\section{Conclusiones}

Acorde a los resultados obtenidos en la investigación, se puede concluir que el pronóstico para las variables analizadas almacenaje y tiempo es muy satisfactorio, ya que los resultados oscilan de la media hacia arriba, con una fuerte ponderación respecto al conjunto de variables analizadas, mientras que para las variables transporte y costo, el pronóstico va de regular a satisfactorio. Por su parte, aduanas es la variable que recibió el pronóstico peor calificado, con valores altos en opciones como: "muy malo". Dichos resultados dejan entrever la fuerte necesidad de mejorar la cadena logística a fin de que el servicio sea vislumbrado como eficiente y competitivo, no obstante es el gobierno quien debe ser el primer impulsor puesto que, se podría afirmar, en términos de los resultados obtenidos, que es la aduana la que muestra la más baja calificación respecto a los servicios logísticos que se ofrecen en el puerto.

Es también importante señalar que la aduana mexicana está pasando por una serie de cambios tanto en su legislación como en la parte operativa, lo que implicaría a mediano y largo plazo una mejora en la administración aduanera, si estas modificaciones se implementan de forma adecuada. Por lo que resultaría importante realizar nuevamente este tipo de estudios en un futuro.

Cada variable, se analizó a su vez con el objetivo de identificar los principales elementos de cada uno de estos cinco factores clave: aduanas, costos, tiempo, almacenaje y transporte, de la cadena logística del comercio exterior del puerto de Lázaro Cárdenas y el grado de importancia de los mismos, respecto del factor considerado como variable dependiente.

En el caso de las aduanas o administración aduanera, según los resultados, se llega: a la conclusión que es necesario brindar más apoyos gubernamentales a fin de incentivar el comercio exterior de México, además de implementar medidas de agilización de trámites para que el tiempo en el despacho aduanero sea menor. Dichas medidas pueden ser: una mayor capacitación, el uso de medios electrónicos de forma intensiva siempre y cuando funcionen de manera eficiente y la incorporación de tecnologías que permitan una revisión rápida y eficaz.

Respecto al almacenaje de mercancía, se podría pensar que la colindancia con la aduana juega un papel fundamental, sin embargo, no es tan importante como el adecuado manejo de las mercancías y el equipo con el que dispongan los encargados del almacén. Por lo que, si se busca mejorar en este apartado de la cadena logística, es de suma importancia implementar tecnologías, acorde con las necesidades de almacenamiento, así como capacitación continua de los recursos humanos, con la finalidad de elevar los niveles de calidad.

En el caso de la variable tiempo, se encontró que no es tan importante el tiempo transcurrido en el proceso de traslado como lo es el cumplimiento de los plazos estipulados, sin embargo, ambos están fuertemente vinculados con la planeación a lo largo de la cadena logística, por lo que la demora o incluso la llegada anticipada de los productos, puede representar un costo adicional (almacenaje) o incluso la pérdida del cliente; lo que implica una pérdida de mercado y disminución de la competitividad del producto frente a sus productos competidores.

Respecto a las variables transporte y costos, se recomienda una mejora en los servicios que actualmente se ofrecen, puesto que el costo está directamente relacionado con el servicio. Por su parte las rutas fiscales establecidas actualmente necesitan una mejora en su infraestructura a su vez es necesario una planeación respecto a la implementación y mejora de nuevas rutas, para contar con mejores corredores logísticos de vanguardia que efectivamente cubran las necesidades de los mercados internacionales. 
Para finalizar, se resalta la importancia que representa el enlace de los operadores a fin de lograr encadenamientos productivos, optimizando la operación de los servicios de manera global y local. Local, mediamente la mejora de los procesos internos de cada proveedor de servicios logísticos; y, global, no como la sumatoria de cada una de las unidades que componen los servicios, sino como el conjunto de la cadena de producción y sus diversas interrelaciones.

\section{Referencias}

APM Terminals, (2018). APM Terminals Lifting Global Trade: http://www.apmterminals.com/news/lazaro-cardenas Banco Mundial (2014). Informe del índice de desempeño logístico. Washington.

Figueredo, G. \& Ballesteros, A. (2016). Identificación del estado de madurez de las frutas con redes neuronales artificiales, una revisión. Revista Ciencia y Agricultura. 13 (1),117-132.

Freeman, J.A.; Skapura, DM. (1993). Redes Neuronales. Algoritmos, aplicaciones y técnicas de propagación. México: Addison-Wesley. 1993; 306 p.

Fugazza M \& Hoffmann J (2017). Liner shipping connectivity as determinant of trade. Journal of Shipping and Trade 2(1). Disponible en: https://doi.org/10.1186/s41072-017-0019-5

Hoffmann J, Wilmsmeier G \& Lun V (2017). Connecting the world through global shipping networks. Journal on Shipping and Trade 2(2),1-4. https://doi.org/10.1186/s41072-017-0020-z

J. Fogarty, R. Baker, S. Hudson (2005). "Case studies in the use of ROC curve analysis for sensor-based estimates in human computer interaction”. ACM International Conference Proceeding Series, Proceedings of Graphics Interface 2005. Waterloo, Ontario, Canada: Canadian Human-Computer Communications Society.

Montaño, J.J. (2002). Redes Neuronales Artificiales aplicadas al análisis de datos. Universitat de Les Illes Balears. Facultad de Psicología. Palma de Mallorca.

Organización Mundial de Comercio (2015). La facilitación del Comercio. Geneva.

Organización Mundial de Comercio (2017). Aid for Trade at a Glance 2017, Promoting Trade, Inclusiveness and Connectivity for Sustainable Development. Geneva.

Rodríguez, P. \& Turias, I. (2016). Una Comparativa entre redes neuronales artificiales y métodos clásicos para la predicción de la movilidad entre zonas de transporte. Aplicación ene 1 Campo de Gibraltar, España. DYNA, 84 (200),209-216.

Secretaría de Comunicaciones y Transportes, SCT, (2017). Secretaría de Comunicaciones y Transportes. Administración Portuaria Integral de Lázaro Cárdenas. Disponible en: http://www.puertolazarocardenas.com.mx/plc25/ ubicacicerca-del-puerto-55

Secretaría de Comunicaciones y Transportes, SCT, (2016). Secretaría de Comunicaciones y Transportes. Distancias. Disponible en: http://www.puertolazarocardenas.com.mx/plc25/distancias

Secretaría de Comunicaciones y Transportes (2013). Programa sectorial de Comunicaciones y Transportes. Programa Nacional de Desarrollo 2013-2018. Gobierno de la República. Ciudad de México.

Sotolongo, G; Suárez, CA.; Guzmán, MV. (2000). Modular Bibliometrics Information System with Propietary Software (MOBIS-ProSoft): a versatile approach to bibliometric research tools. Library and Information Science Electronic Journal (LIBRES). 2000; 10(2): http://libres.curtin.edu.au/

Sotolongo, G. \& Guzmán, M.V. (2001). Aplicaciones de las redes neuronales. El caso de la Biliometría. Ciencias de la Información. 32 (1), 27-34.

United Nations Conference on Trade and Development, UNTACD, (2017). Review of Maritime Transport. United Nations, New York and Geneva.

Widrow, B. \& Hoff, M. (1960). Adaptive switching circuits. En J. Anderson y E. Rosenfeld (Eds.), Neurocomputing (pp. 126-134). Cambridge, Mass.: The MIT.

Wilmsmeier G, Gonzalez-Aregall M \& Spengler T (2017). The liner shipping industry: Looking beyond firms - Markets structure, competition and concentration. Annual Conference of the International Association of Maritime Economists. 27-30 June. Kyoto. 


\section{Anexo. Pruebas de confiabilidad, resumen del modelo}

Resumen del modelo

\begin{tabular}{|ll|l|}
\hline Entrenamiento & Error de entropía cruzada & 47.420 \\
& $\begin{array}{l}\text { Porcentaje de pronósticos } \\
\text { incorrectos }\end{array}$ & $32.6 \%$ \\
& Regla de parada utilizada & $\begin{array}{l}\text { 1 pasos consecutivos sin } \\
\text { disminución del error }\end{array}$ \\
& Tiempo de preparación & $0: 00: 00.03$ \\
\hline Pruebas & $\begin{array}{l}\text { Error de entropía cruzada } \\
\text { Porcentaje de pronósticos } \\
\text { incorrectos }\end{array}$ & $\begin{array}{l}19.747 \\
\end{array}$ \\
\hline
\end{tabular}

Variable dependiente: Aduana

a. Los cálculos de error se basan en la muestra de comprobación.

Resumen del modelo

\begin{tabular}{|ll|l|}
\hline Entrenamiento & Error de entropía cruzada & 40.987 \\
& $\begin{array}{l}\text { Porcentaje de pronósticos } \\
\text { incorrectos }\end{array}$ & $18.8 \%$ \\
& Regla de parada utilizada & $\begin{array}{l}1 \text { pasos consecutivos sin } \\
\text { disminución del error }\end{array}$ \\
& Tiempo de preparación & $0: 00: 00.03$ \\
\hline Pruebas & $\begin{array}{l}\text { Error de entropía cruzada } \\
\text { Porcentaje de pronósticos } \\
\text { incorrectos }\end{array}$ & 9.371 \\
& & $12.5 \%$ \\
\hline
\end{tabular}

Variable dependiente: Almacenaje

a. Los cálculos de error se basan en la muestra de comprobación.

Resumen del modelo

\begin{tabular}{|ll|l|}
\hline Entrenamiento & Error de entropía cruzada & 37.154 \\
& $\begin{array}{l}\text { Porcentaje de pronósticos } \\
\text { incorrectos }\end{array}$ & $20.8 \%$ \\
& Regla de parada utilizada & $\begin{array}{l}1 \text { pasos consecutivos sin } \\
\text { disminución del error }\end{array}$ \\
& Tiempo de preparación & $0: 00: 00.03$ \\
\hline Pruebas & $\begin{array}{l}\text { Error de entropía cruzada } \\
\text { Porcentaje de pronósticos } \\
\text { incorrectos }\end{array}$ & 14.872 \\
& & $16.8 \%$ \\
\hline
\end{tabular}

Variable dependiente: Tiempo

a. Los cálculos de error se basan en la muestra de comprobación. 
Resumen del modelo

\begin{tabular}{|ll|l|}
\hline Entrenamiento & Error de entropía cruzada & 36.284 \\
& $\begin{array}{l}\text { Porcentaje de pronósticos } \\
\text { incorrectos }\end{array}$ & $20.0 \%$ \\
& Regla de parada utilizada & $\begin{array}{l}1 \text { pasos consecutivos sin } \\
\text { disminución del error }\end{array}$ \\
& Tiempo de preparación & $0: 00: 00.03$ \\
\hline Pruebas & $\begin{array}{l}\text { Error de entropía cruzada } \\
\text { Porcentaje de pronósticos } \\
\text { incorrectos }\end{array}$ & 17.233 \\
& & $12.5 \%$ \\
\hline
\end{tabular}

Variable dependiente: Transporte

a. Los cálculos de error se basan en la muestra de comprobación. 